\title{
Identification of Cropping System Module for Irrigated Farming System of Rewa Region
}

\author{
Jugnahake Monica, Prajapat Rohit, B.M. Maurya and S.M. Kurmvanshi*
}

All India Coordinated Research Project on Integrated Farming System, College of Agriculture, JNKVV, Rewa (M.P) 486001, India

*Corresponding author

\section{A B S T R A C T}

\begin{tabular}{|l|}
\hline K e y w or d s \\
Cropping system, Rewa \\
region, Rice-wheat
\end{tabular}

\section{Introduction}

Rice is an important crop of rice-wheat crop zone of Madhya Pradesh which occupies an area of 16.75 lakh hectares. The average productivity is $1194 \mathrm{~kg} / \mathrm{ha}$. In Rewa region of Madhya Pradesh, rice is cultivated in 6.09 lakh hectares with the production of 4.47 lakh tonnes and the average productivity of rice is $917 \mathrm{~kg} / \mathrm{ha}$. It is very low as compared to national and state average productivity (Rao 2012). It is due use of local varieties, erratic and uneven distribution of rains as well as prolong dry spells observed frequently.

Introduction of pulses and oil seed in the system are more beneficial than cereal-cereal sequence (Umarani et al., 1992). In cropping system inclusion of pulses, oilseed and vegetables are more beneficial than cereals after cereals (Kumpawat, 2001). Rice is predominated crop in Rewa region of Madhya Pradesh. It is difficult to replace the rice by any other crop in rainy season due to soil and climatic condition. Hence, only option left is to replace the wheat and gram crop in winter season by mustard, barley, potato, toria, onion, garlic, pea, pea + mustard and berseem.

Experimental evidences show that rice-wheat, rice-green manure-barley, rice-gram + mustard-green manure, rice-pea-green gram, rice-potato-green gram, rice-pea + mustardgreen manure, rice-berseem, rice-barley fodder-bajra fodder, rice-garlic and rice-toriaonion have been found effective and 
remunerative cropping sequence in different parts of the country.

Rice-potato-wheat system utilizes the water most efficiently closely followed by medium duration rice-berseem system. The growing of vegetable pea and potato between rice and wheat increased the organic carbon by $8.9 \%$ to $17.1 \%$, available $\mathrm{N}$ by $6.3 \%$ and $8.3 \%$ Olsen's production by $6.3 \%$ and $19 \%$, respectively in soil as compared to rice-wheat sequence (Kharub et al., 2003).

Development of soil sickness for rice and wheat crop has also been observed due to continuous cropping (Kharub et al., 2003). The soil productivity is declining day by day because both crops are exhaustive (Choudhary et al., 2007). The rice followed legume crop sequence has the ability to ameliorate the soil fertility by fixing atmospheric nitrogen. Different rabi crops have some residual effect on succeeding crops. Annual report (2010-11) of All India Coordinated Research Project on IFS Modipuram indicates that rice yield was maximum when rice was grown after wheatdhaincha and mustard as compared to ricewheat cropping system at Varanasi (U.P). The grain yield of rice was $11.58 \%$ higher when grown after wheat-dhaincha and $10.86 \%$ higher when rice was grown after mustardgreen gram.

\section{Materials and Method}

The present field investigation was made under all India coordinated research project on farming system at Kuthulia farm of JNKVV, college of agriculture Rewa (M.P.) during kharif season of 2017-18 in which ten cropping system (rice-wheat, rice-green manure-barley, rice-gram + mustard-green manure, rice-pea-green gram, rice-potatogreen gram, rice-pea + mustard-green manure, rice-berseem, rice-barley fodder-bajra fodder, rice-garlic and rice-toria-onion) were taken.
The rice variety was Danteshwari and PS-5 (rice-berseem and rice-garlic). The experiment was started in the year 2007-08 and same layout was adopted for present study. The experimental design was RBD with three replications.

The soil of experimental field was silty clay loam in texture, neutral in reaction (soil $\mathrm{pH}$ $7.10)$, low in organic carbon $(0.39 \%)$, low in available nitrogen $(163.83 \mathrm{~kg} / \mathrm{ha})$, medium in available phosphorus (16.33 $\mathrm{kg} / \mathrm{ha})$ and available potash was $(219.7 \mathrm{~kg} / \mathrm{ha})$. The total rainfall during crop season was $856.9 \mathrm{~mm}$ received in 42 rainy days. The dry spells were observed in month of September to October.

The rice crop was transplanted on 06/07/2017 at planting geometry of $20 \mathrm{~cm} \times 15 \mathrm{~cm}$ in which two seedling per hill was transplanted. The fertilizer dose for rice crop was $120 \mathrm{~kg} \mathrm{~N}, 60 \mathrm{~kg}$ $\mathrm{P}_{2} \mathrm{O}_{5}$, and $40 \mathrm{~kg} \mathrm{~K}_{2} \mathrm{O} / \mathrm{ha}$ under all cropping system. All the recommended package of practices were adopted for the irrigated condition. The rice variety Danteshwari was harvested on 27/09/2017 and PS-5 on $08 / 10 / 2017$. After harvest of kharif crops different rabi crops were sown on $1^{\text {st }}$ week of October. Garlic and berseem were sown on $19^{\text {th }}$ October while wheat crop was sown on $24^{\text {th }}$ November in 2017-18.

\section{Results and Discussion}

\section{Residual effect on rice}

The yield contributing character of rice under the residual effect of different cropping systems has been given in Table 1. After perusal of results it is evident that yield contributing characters of rice were influenced significantly. It is clear from the result that yield attributing characters were superior in rice variety Danteshwari grown after Ricegram + mustard-green manure, rice-barleybajra and rice-toria-onion cropping system as 
compared to existing rice-wheat system. The number of productive tillers per meter row length was significantly maximum in ricegram + mustard-green manure and rice-toriaonion cropping system. These cropping systems gave 13.75 to $14.32 \%$ higher number of productive tillers $/ \mathrm{m}^{2}$ as compared to existing rice-wheat system. Productive tillers $/ \mathrm{m}^{2}$ were higher by $10.3 \%$ in rice-green manure-barley, $12.42 \%$ in rice-barley-bajra and $7.32 \%$ in rice-potato-green gram cropping systems over rice-wheat existing cropping system. The number of sound grains and grain yield/panicle were maximum in rice-garlic and rice-gram + mustard-green manure cropping systems. These cropping systems gave more than $20 \%$ higher number of sound grains and grain yield/panicle as compared to existing rice-wheat system. Rice variety PS-5 gave higher yield/panicle in rice-garlic than riceberseem cropping system. It may be due to positive residual effect of preceding rabi crops on succeeding rice. It may be due to enhancement of organic carbon in soil by $25 \%$ to $51 \%$ and potash as compared to initial status. The crop like mustard, garlic, toriaonion, green manure-barley grown in rabi and summer have good residual and cumulative effect on succeeding rice by providing favorable soil atmosphere and enhancing the organic carbon and potassium by which growth of rice were increased in these cropping systems (Table 3). These findings are in conformity with the findings of Maurya et al., (2011), Dhakad (2013), Yadav et al., (2014) and Chouriya (2016).

The data on grain yield of rice under the residual effect of different cropping systems is given in Table 2 reveals that rice yield was increased by $7.19 \%$ in rice-pea-green gram, $8.45 \%$ in rice-green manure-barley, $9.3 \%$ in rice-potato-green gram, $13.42 \%$ in barley fodder-bajra fodder and $11.71 \%$ in rice-toriaonion cropping systems as compared to existing rice-wheat cropping system. Rice variety PS-5 gave $8.48 \%$ lower grain yield in rice-berseem and $16.36 \%$ in rice-garlic cropping systems as compared to rice variety Danteshwari in rice-wheat system. It may be due to positive residual effect of preceding rabi and summer crops like green manurebarley, gram + mustard-green manure, potatogreen gram, barley-bajra and toria-onion by which growth and yield contributing characters of rice were increased significantly. The yield contributing character as number of productive tillers $/ \mathrm{m}^{2}$, number of sound grains/ panicle and grain yield per panicle were increased significantly as compared to rice grown after wheat. It may be due to amelioration of organic matter in soil by green manure-barley, gram + mustard-green manure, pea-green gram, potato-green gram, barleybajra and toria-onion cropping system. The present experiment was started in the year 2006-07, therefore, there was buildup of residual effect by different rabi and summer crops. The positive effect of preceding berseem, pea-wheat, mustard-green manure, potato-wheat and toria-onion on rice were also reported by Sharma and Jain (1997), Upadhyay (2007), Yadav et al., (2014) and Chouriya (2016).

\section{Performance of different rabi crops and cropping systems}

Data pertaining to economical yield of different rabi and summer crops have been given in Table 2. It is clear from the result that garlic crop performed better after rice followed by gram + mustard intercropping in $3: 1$. These crops were found superior than existing wheat in rabi. Rice equivalent yield under different cropping systems has been given in Table 2 reveals that rice-garlic cropping system gave maximum rice equivalent yield $195.64 \mathrm{q} / \mathrm{ha}$ followed by ricepotato-green gram (165.06 q/ha) and intercropping of gram + mustard in $3: 1$ (151.52 q/ha). 
Table.1 Yield contributing character of rice as influenced by residual effect of different cropping system

\begin{tabular}{|c|c|c|c|c|c|c|c|}
\hline Treatment & $\begin{array}{l}\text { Plant } \\
\text { height } \\
(\mathrm{cm})\end{array}$ & $\begin{array}{l}\text { Number of } \\
\text { productive } \\
\text { tillers } / \mathrm{m}^{2} \text { at } \\
\text { harvest }\end{array}$ & $\begin{array}{l}\text { Panicle } \\
\text { length } \\
\text { (cm) }\end{array}$ & $\begin{array}{l}\text { Weight } \\
\text { of } \\
\text { panicle } \\
\text { (g) }\end{array}$ & $\begin{array}{c}\text { Number of sound } \\
\text { grains/panicle at } \\
\text { harvest }\end{array}$ & $\begin{array}{l}\text { Grain } \\
\text { yield/panicle } \\
\text { (g) }\end{array}$ & $\begin{array}{l}\text { Test } \\
\text { weight } \\
\text { (g) }\end{array}$ \\
\hline $\begin{array}{l}\text { T }_{1} \text { Rice(Danteshwari)- } \\
\text { Wheat(HD-2864) }\end{array}$ & 60.99 & $348.88(0.00)$ & 23.7 & 2.59 & $89.00(0.00)$ & $2.22(0.00)$ & 25.38 \\
\hline $\begin{array}{l}\mathrm{T}_{2} \text { Rice(Danteshwari)- Green } \\
\text { manure-barley(Gitanjali) }\end{array}$ & 66.99 & $384.98(10.34 \%)$ & 23.14 & 2.64 & $96.33(8.23 \%)$ & $2.40(8.10 \%)$ & 25.33 \\
\hline $\begin{array}{l}\mathrm{T}_{3} \text { Rice(Danteshwari)-Gram(JG- } \\
\text { 322)+Mustard(Pusa bold)-Green } \\
\text { manure }\end{array}$ & 64.68 & $398.86(14.32 \%)$ & 22.29 & 2.45 & $107.00(20.22 \%)$ & $2.67(20.27 \%)$ & 25.90 \\
\hline $\begin{array}{l}\text { T}_{4} \text { Rice(Danteshwari)- } \\
\text { Pea(Arkel)-Green gram(PDM- } \\
\text { 139) }\end{array}$ & 62.55 & $363.66(4.23 \%)$ & 23.24 & 2.56 & $88.00(-1.12 \%)$ & $2.20(-0.90 \%)$ & 25.16 \\
\hline $\begin{array}{l}\text { T}_{5} \text { Rice(Danteshwari)- } \\
\text { Potato(Kufri chandramukhi)- } \\
\text { Green gram(PDM-139) }\end{array}$ & 61.38 & $374.43(7.32 \%)$ & 22.55 & 2.33 & $81.33(8.61 \%)$ & $1.95(-12.16 \%)$ & 24.71 \\
\hline $\begin{array}{l}\text { T}_{6} \text { Rice(Danteshwari)- } \\
\text { Pea(Arkel)+Mustard(pusa bold)- } \\
\text { Green manure }\end{array}$ & 62.94 & $352.60(1.06 \%)$ & 23.77 & 2.38 & $79.86(-10.26 \%)$ & $1.97(-11.26 \%)$ & 24.78 \\
\hline $\mathrm{T}_{7}$ Rice(PS-5)-Berseem(JB-1) & 74.55 & $371.10(6.36 \%)$ & 27.09 & 3.12 & $92.00(3.37 \%)$ & $2.57(15.76 \%)$ & 28.90 \\
\hline $\begin{array}{l}\text { T8 Rice(Danteshwari)-Barley } \\
\text { fodder(JB-58)-Bajra } \\
\text { fodder(WCC-75) }\end{array}$ & 65.33 & $392.22(12.42 \%)$ & 23.59 & 2.72 & $103.00(15.73 \%)$ & $2.75(23.87 \%)$ & 26.46 \\
\hline T9 $_{9} \operatorname{Rice}(\mathbf{P S}-5) \operatorname{Garlic}(\mathrm{G}-1)$ & 68.83 & $356.49(2.18 \%)$ & 25.09 & 3.10 & $107.33(20.59 \%)$ & $2.99(34.87 \%)$ & 28.00 \\
\hline $\begin{array}{l}\text { T10 Rice(Danteshwari)-Toria(T- } \\
\text { 9)-Onion(AFLR) }\end{array}$ & 63.38 & $396.88(13.75 \%)$ & 21.23 & 2.39 & $87.86(-2.24 \%)$ & $2.28(2.70 \%)$ & 26.29 \\
\hline S.Em+ & 2.08 & 0.68 & 0.58 & 0.17 & 2.99 & 0.17 & 1.74 \\
\hline CD at $5 \%$ & 2.14 & 25.68 & 1.70 & 0.48 & 8.84 & 0.48 & NS \\
\hline
\end{tabular}

Figures in parentheses are $\%$ increase or decrease over $\mathrm{T}_{1}$ (rice-wheat existing cropping system) 
Table.2 Grain yield, REY, gross and net profit and B: C ratio of different cropping systems

\begin{tabular}{|c|c|c|c|c|c|c|c|}
\hline \multirow[t]{2}{*}{ Treatment } & \multicolumn{3}{|c|}{ Yield q/ha } & \multirow[t]{2}{*}{ REY } & \multirow{2}{*}{$\begin{array}{l}\text { Gross } \\
\text { return } \\
\text { Rs/ha }\end{array}$} & \multirow{2}{*}{$\begin{array}{l}\text { Net return } \\
\text { Rs/ha }\end{array}$} & \multirow[t]{2}{*}{ B:C ratio } \\
\hline & Kharif & Rabi & $\begin{array}{c}\text { Rabi/ } \\
\text { Summer }\end{array}$ & & & & \\
\hline $\begin{array}{l}\text { T }_{1} \text { Rice (Danteshwari)- Wheat(HD- } \\
\text { 2864) }\end{array}$ & 50.29 & 55.53 & & 117.67 & 211815 & 133613 & 2.7 \\
\hline $\begin{array}{l}T_{2} \text { Rice (Danteshwari)- Green manure- } \\
\text { barley (Gitanjali) }\end{array}$ & $\begin{array}{c}54.54 \\
(8.45 \%)\end{array}$ & 146.6 & 56.62 & $\begin{array}{c}129.73 \\
(10.24 \%)\end{array}$ & 233520 & $\begin{array}{l}161335 \\
(20.74 \%)\end{array}$ & 3.23 \\
\hline $\begin{array}{l}\text { T3 Rice (Danteshwari)-Gram (JG- } \\
\text { 322)+Mustard (Pusa bold)-Green } \\
\text { manure }\end{array}$ & $\begin{array}{c}56.62 \\
(12.58 \%)\end{array}$ & $\begin{array}{l}13.43 \\
23.23\end{array}$ & 125.9 & $\begin{array}{c}151.52 \\
(28.76 \%)\end{array}$ & 272739 & $\begin{array}{c}201229 \\
(50.60 \%)\end{array}$ & 3.81 \\
\hline $\begin{array}{l}\text { T }_{4} \text { Rice (Danteshwari)-Pea (Arkel)- } \\
\text { Green gram (PDM-139) }\end{array}$ & $\begin{array}{c}53.91 \\
(7.19 \%)\end{array}$ & 82.14 & 9.16 & $\begin{array}{c}117.66 \\
(0.00)\end{array}$ & 211804 & $\begin{array}{l}139774 \\
(4.61 \%)\end{array}$ & 2.94 \\
\hline $\begin{array}{l}\text { T}_{5} \text { Rice (Danteshwari)-Potato (Kufri } \\
\text { chandramukhi)- Green gram (PDM- } \\
\text { 139) }\end{array}$ & $\begin{array}{c}54.97 \\
(9.30 \%)\end{array}$ & 219.23 & 7.86 & $\begin{array}{c}165.06 \\
(40.27 \%)\end{array}$ & 297112 & $\begin{array}{c}170327 \\
(27.47 \%)\end{array}$ & 2.34 \\
\hline $\begin{array}{l}\mathrm{T}_{6} \text { Rice (Danteshwari)-Pea } \\
\text { (Arkel)+Mustard (pusa bold)- Green } \\
\text { manure }\end{array}$ & $\begin{array}{c}50.63 \\
(0.67 \%)\end{array}$ & $\begin{array}{l}19.00 \\
15.05\end{array}$ & 116.8 & $\begin{array}{l}124.33 \\
(5.65 \%)\end{array}$ & 223808 & $\begin{array}{l}153423 \\
(14.82 \%)\end{array}$ & 3.17 \\
\hline T $_{7}$ Rice(PS-5)-Berseem (JB-1) & $\begin{array}{c}45.84 \\
(-8.84 \%)\end{array}$ & 751.73 & 1.5 & $\begin{array}{c}128.78 \\
(9.44 \%)\end{array}$ & 231804 & $\begin{array}{c}160789 \\
(20.33 \%)\end{array}$ & 3.26 \\
\hline $\begin{array}{l}\text { T8 Rice (Danteshwari)-Barley fodder } \\
\text { (JB-58)-Bajra fodder (WCC-75) }\end{array}$ & $\begin{array}{c}57.04 \\
(13.42 \%)\end{array}$ & 458.73 & 502.33 & $\begin{array}{c}98.80 \\
(-16.03 \%)\end{array}$ & 177849 & $\begin{array}{c}115284 \\
(-13.71 \%)\end{array}$ & 2.84 \\
\hline T9 Rice (PS-5) Garlic (G-1) & $\begin{array}{c}42.06 \\
(-16.36 \%)\end{array}$ & 131.55 & & $\begin{array}{c}195.64 \\
(66.26 \%)\end{array}$ & 352153 & $\begin{array}{c}211188 \\
(58.05 \%)\end{array}$ & 2.49 \\
\hline $\begin{array}{l}\text { T10 Rice (Danteshwari)-Toria (T-9)- } \\
\text { Onion (AFLR) }\end{array}$ & $\begin{array}{c}56.18 \\
(11.71 \%)\end{array}$ & 7.86 & 173.5 & $\begin{array}{c}148.69 \\
(26.36 \%)\end{array}$ & 267648 & $\begin{array}{c}121959 \\
(-8.72 \%)\end{array}$ & 1.97 \\
\hline S.Em+ & 1.13 & & & 2.50 & & & \\
\hline CD at $5 \%$ & 3.13 & & & 7.40 & & & \\
\hline
\end{tabular}

Figures in parentheses are \% increase or decrease over rice-wheat systems. 
Table.3 Chemical properties of soil in different cropping system

\begin{tabular}{|c|c|c|c|c|c|c|}
\hline \multirow[t]{2}{*}{ Treatment } & \multirow[t]{2}{*}{ pH } & \multirow{2}{*}{$\begin{array}{c}\text { Ec } \\
\text { Mmhos } / \mathrm{cm}^{2}\end{array}$} & \multirow[t]{2}{*}{ Oc g/kg } & \multicolumn{3}{|c|}{ Available nutrient kg/ha } \\
\hline & & & & $\mathbf{N}$ & $\mathbf{P}$ & $\mathbf{K}$ \\
\hline $\begin{array}{l}\text { T } 1 \text { Rice (Danteshwari)- Wheat (HD- } \\
\text { 2864) }\end{array}$ & 6.52 & 0.39 & $\begin{array}{c}4.9 \\
(25.6 \%)\end{array}$ & $\begin{array}{c}145.9 \\
(-10.94 \%)\end{array}$ & $\begin{array}{c}11.9 \\
(-27.12 \%)\end{array}$ & $\begin{array}{c}315.8 \\
(43.70 \%)\end{array}$ \\
\hline $\begin{array}{l}T_{2} \text { Rice (Danteshwari)- Green manure- } \\
\text { barley (Gitanjali) }\end{array}$ & 6.61 & 0.42 & $\begin{array}{c}5.2 \\
(33.33 \%)\end{array}$ & $\begin{array}{c}139.9 \\
(-14.60 \%)\end{array}$ & $\begin{array}{c}11.5 \\
(-29.57 \%)\end{array}$ & $\begin{array}{c}319.9 \\
(45.56 \%)\end{array}$ \\
\hline $\begin{array}{l}T_{3} \text { Rice (Danteshwari)-Gram (JG- } \\
\text { 322)+Mustard (Pusa bold)-Green } \\
\text { manure }\end{array}$ & 6.72 & 0.48 & $\begin{array}{c}4.9 \\
(25.64 \%)\end{array}$ & $\begin{array}{c}142.7 \\
(-12.89 \%)\end{array}$ & $\begin{array}{c}12.6 \\
(-22.84 \%)\end{array}$ & $\begin{array}{c}316.9 \\
(44.20 \%)\end{array}$ \\
\hline $\begin{array}{l}\text { T}_{4} \text { Rice (Danteshwari)-Pea (Arkel)- } \\
\text { Green gram (PDM-139) }\end{array}$ & 6.90 & 0.51 & $\begin{array}{c}5.0 \\
(28.20 \%)\end{array}$ & $\begin{array}{c}145.3 \\
(-11.31 \%)\end{array}$ & $\begin{array}{c}13.7 \\
(-16.10 \%)\end{array}$ & $\begin{array}{c}320.7 \\
(45.95 \%)\end{array}$ \\
\hline $\begin{array}{l}T_{5} \text { Rice (Danteshwari)-Potato (Kufri } \\
\text { chandramukhi)- Green gram (PDM-139) }\end{array}$ & 7.11 & 0.49 & $\begin{array}{c}5.1 \\
(30.76 \%)\end{array}$ & $\begin{array}{c}142.9 \\
(-12.77 \%)\end{array}$ & $\begin{array}{c}14.1 \\
(-13.65 \%)\end{array}$ & $\begin{array}{c}321.1 \\
(46.11 \%)\end{array}$ \\
\hline $\mathrm{T}_{7} \operatorname{Rice}(\mathrm{PS}-5)$-Berseem (JB-1) & 6.63 & 0.28 & $\begin{array}{c}5.1 \\
(30.76 \%)\end{array}$ & $\begin{array}{c}130.1 \\
(-20.58 \%)\end{array}$ & $\begin{array}{c}17.2 \\
(5.32 \%)\end{array}$ & $\begin{array}{c}220.1 \\
(0.15 \%)\end{array}$ \\
\hline $\begin{array}{l}\mathrm{T}_{8} \text { Rice (Danteshwari)-Barley } \\
\text { fodder(JB-58)-Bajra fodder(WCC-75) }\end{array}$ & 6.29 & 0.49 & $\begin{array}{c}5.9 \\
(51.28 \%)\end{array}$ & $\begin{array}{c}129.8 \\
(-20.77 \%)\end{array}$ & $\begin{array}{c}15.8 \\
(-3.24 \%)\end{array}$ & $\begin{array}{c}311.2 \\
(41.60 \%)\end{array}$ \\
\hline T, Rice (PS-5) Garlic (G-1) & 6.86 & 0.42 & $\begin{array}{c}5.8 \\
(48.71 \%)\end{array}$ & $\begin{array}{c}146.2 \\
(-10.76 \%)\end{array}$ & $\begin{array}{c}16.1 \\
(-1.40 \%)\end{array}$ & $\begin{array}{c}315.3 \\
(43.47 \%)\end{array}$ \\
\hline $\begin{array}{l}\text { T10 Rice (Danteshwari)-Toria (T-9)- } \\
\text { Onion (AFLR) }\end{array}$ & 6.71 & 0.47 & $\begin{array}{c}5.6 \\
(43.58 \%)\end{array}$ & $\begin{array}{c}169.5 \\
(3.46 \%)\end{array}$ & $\begin{array}{c}13.2 \\
(-19.16 \%)\end{array}$ & $\begin{array}{c}228.3 \\
(3.88 \%)\end{array}$ \\
\hline Initial & 7.10 & 0.53 & 3.9 & 163.83 & 16.33 & 219.76 \\
\hline
\end{tabular}

Figures in parentheses are \% increase (+) or decrease (-) over initial status. 
Rice-barley-bajra cropping system was found inferior than existing rice-wheat system.

Net profit and $\mathrm{B}: \mathrm{C}$ ratio have been given in Table 2 make it clear that maximum net profit Rs 211188/ha was obtained in rice-garlic cropping system followed by Rs 201229/ha in rice-gram + mustard intercropping in 3:1. These cropping systems gave $50.6 \%$ to $58.05 \%$ higher net profit than existing ricewheat system. It may be due to higher productivity of these crops having more market rate. Similar findings were also reported by Maurya et al., (2011) and Choriya et al., (2016).

\section{Effect on chemical properties of soil}

The chemical properties of soil after completion of 14 crop cycle have been given in Table 3 reveals that soil $\mathrm{pH}$ was reduced in rice-barley-bajra and rice-wheat cropping system as compared to initial status. Electrical conductivity of soil was constant. Organic carbon status was increased in all cropping system by 25 to $51 \%$ and maximum organic carbon was noted in rice-barley-bajra cropping system. The available nitrogen and phosphorus status were decreased while available potash status was increased considerably under different cropping system.

On the basis of above it has been concluded that rice-garlic, rice-gram + mustard intercropping in 3:1 and rice-potato-green gram cropping system were better than existing rice-wheat cropping system in Rewa region of Madhya Pradesh under irrigated condition.

\section{References}

Chouriya S R.2016. Residual effect of different rabi crops on succeeding rice under irrigated condition in Kymore Plateau and Satpura Hills agro climatic zone of Madhya Pradesh. M.sc. Agriculture thesis submitted to the JNKVV Jabalpur.

Chouriya SR, Chouhan M, Kurmvanshi SM and Maurya BM. 2016. Performance of different bio-intensive need based cropping system under irrigated condition. Mysore Journal of Agriculture science 50 (4): 716-720.

Dhakad SS.2013.Studies on bio-intensive cropping system based on rice under irrigated conditions of in Kymore Plateau of Madhya Pradesh. M.sc. Agriculture thesis submitted to the JNKVV Jabalpur.

Kharub AS, Chouhan DS, Sharma RK, Chhokar RS and Tripathi SC.2003. Diversification of rice-wheat system for improving soil fertility and productivity. Indian Journal of Agronomy 48 (3): 149-152.

Kumpawat BS.2001. Production potential and economics of different crop sequence. Indian Journal of Agronomy 46 (3): 421-424.

Maurya BM, Upadhyay VB and Mishra Sandhya.2011. Crop diversification based on rice in Kymore Satpura agro climatic zone of Madhya Pradesh. Mysore Journal of Agriculture science 45 (4): 916-917.

Rao SK.2012. Rice knowledge management portal http://www.rkmp.co.in.

Sharma RS and Jain KK.1997. Agronomic research advances in rice-wheat system in Madhya Pradesh. Advances in Agricultural research in India 7: 139157.

Umarani NK, Gaikwad CB and Gore BN.1992. Sustainability of cropping systems under dry land condition. Indian Journal of Agronomy 37(4): 645649.

Upadhyay VB, Jain Vikash, Vishwakarma SK and Kumar Ashok K.2007. Diversification of rice based cropping 
system for Kymore Plateau and Satpura Hills zone of Madhya Pradesh. Sustainable agricultural production, extended summaries, $3^{\text {rd }}$ national symposium on integrated farming system. P: 128-130.
Yadav PS, Maurya BM and Kurmvanshi SM.2014. Growth and development of rice under different irrigated cropping system. Mysore Journal of Agriculture science 48(3): 451-455.

\section{How to cite this article:}

Jugnahake Monica, Prajapat Rohit, B.M. Maurya and Kurmvanshi, S.M. 2018. Identification of Cropping System Module for Irrigated Farming System of Rewa Region. Int.J.Curr.Microbiol.App.Sci. 7(10): 687-694. doi: https://doi.org/10.20546/ijcmas.2018.710.076 